\title{
Differential Roles of Lipin1 and Lipin2 in the Hepatitis C Virus Replication Cycle ${ }^{\dagger}$
}

\author{
Victoria Castro ${ }^{1}$, Gema Calvo ${ }^{1}$, Ginés Ávila-Pérez ${ }^{1}$, Marlène Dreux ${ }^{2}$ and Pablo Gastaminza ${ }^{1, *}$ \\ 1 Department of Cellular and Molecular Biology Centro Nacional de Biotecnología-Consejo Superior de \\ Investigaciones Científicas, 28049 Madrid, Spain; vcastro@cnb.csic.es (V.C.); gcalvo@cnb.csic.es (G.C.); \\ gines_ap@hotmail.com (G.Á.-P.) \\ 2 CIRI, Inserm, U1111, Université Claude Bernard Lyon 1, CNRS, UMR5308, École Normale Supérieure de \\ Lyon, Univ Lyon, F-69007 Lyon, France; marlene.dreux@ens-lyon.fr \\ * Correspondence: pgastaminza@cnb.csic.es \\ † Presented at Viruses 2020-Novel Concepts in Virology, Barcelona, Spain, 5-7 February 2020.
}

Published: 9 June 2020

\begin{abstract}
Although their origin, nature and structure are not identical, a common feature of positive-strand RNA viruses is their ability to subvert host lipids and intracellular membranes to generate replication and assembly complexes. Recently, lipin1, a cellular enzyme that converts phosphatidate into diacylglycerol, has been involved in the formation of the membranous web that hosts hepatitis $\mathrm{C}$ virus (HCV) replicase. In the liver, lipin1 cooperates with lipin2 to maintain glycerolipid homeostasis. We extended our previous study of the lipin family in HCV infection by determining the impact of the lipin2 silencing on viral replication. In contrast to the specific impact of lipin1 silencing on HCV replication, our data suggest a broader function of lipin2 not only in HCV infection, but also for the replication of other RNA viruses. Moreover, uninfected lipin2- but not lipin1-deficient cells display alterations in mitochondrial and Golgi morphology, suggesting that lipin2 contributes to the maintenance of the overall organelle architecture. Coinciding with Golgi fragmentation, our data reveal that lipin2 silencing mainly interferes with $\mathrm{HCV}$ virion secretion at late stages of the infection without significantly affecting viral replication or assembly. Overall, this study reveals distinctive functions of lipin1 and lipin2 in cells of hepatic origin, a context in which they are often considered functionally redundant.
\end{abstract}

Keywords: hepatitis C virus; lipin family; lipid homeostasis; Golgi apparatus

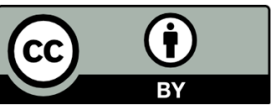

(C) 2020 by the authors. Licensee MDPI, Basel, Switzerland. This article is an open access article distributed under the terms and conditions of the Creative Commons Attribution (CC BY) license (http://creativecommons.org/licenses/by/4.0/). 